\title{
DOS ESTERCULIÁCEAS SUDAMERICANAS NUEVAS
}

\author{
por CARMEN L. CRISTOBAL
}

\begin{abstract}
Summary
Byttneria fontis (sect. Crassipetala) from Nuflo de Chavez province, Santa Cruz, Bolivia and Ayenia latifolia (sect. Ayenia) from Goiás and Bahia, Brasil, are described and illustrated. A comparison with the related species are given.
\end{abstract}

\section{Byttneria fontis Cristóbal nov.sp.} Fig. 1

Frutex scandens; caules aculeati, pilis stellatis, dense pubescentes. Petiolus 6-10 mm longus. Lamina 3-5,5 cm longa $\times 2,5-5 \mathrm{~cm}$ lata, obovata vel suborbicularis, dentata, base cuneata, integra, apice rotundata, supra pilis stellatis sparcis, subtus tomentosa; nectario multiaperturato vena media ad basim disposito. Petala membranacea lamina filiforme, basim versus leviter pubescens. Tubus staminalis membranaceus, staminodia latere externo 3 mamillis instructis. Fructus sphaericus ca. $1 \mathrm{~cm}$ diam. coccis lignosis, indehiscentibus, spisse aculeis conicis obtectus.

Holotipo: BOLIVIA. Santa Cruz: prov. Nuflo de Chavez. Estancia San Miguelito, 200 km NE de Santa Cruz, puesto La Pascana. Bosque semideciduo de llanura, en orilla de río San Julián, $17^{\circ} 45^{\prime} \mathrm{S}, 270 \mathrm{~m}$. Arbusto sarmentoso, $2 \mathrm{~m}$; flores blancas. En sitio encharcado de orilla de río. 14 Mar 95 [en flor], A.F. Fuentes 457 (USZ). Isotipos: CTES,MO.

Arbusto apoyante ca. de $2 \mathrm{~m}$ alt., tallos con indumento denso de pelos estrellados erectos, suaves, uniformes; aguijones recurvados, 2-3 $\mathrm{mm}$ long., pubescentes hasta cerca del ápice, a menudo dispuestos en los nudos o cerca de ellos. Estípulas fugaces, subuladas, ca. 1,5 mm long. Pecíolo terete, 6-10 mm long., con el mismo indumento del tallo o algo más denso, rara vez con algún aguijón pequeño. Lámina foliar

\footnotetext{
${ }^{1}$ Instituto de Botánica del Nordeste, C.C. 209, 3400 Corrientes, Argentina.
}

3-5,5 cm long. x 2,5-5 cm lat., anchamente obovada, también suborbicular, obtusa, base cuneada con los lados rectos y enteros, el resto del margen dentado, dientes distantes, poco profundos, terminados en un pequeño mucrón; nectario 1, en el hipofilo en la base de la vena media, 1-2 mm long., multiaperturado, castaño, brillante, glabro; hipofilo tomentoso, grisáceo, algo más claro que el epifilo, pelos estrellados multiradiados, algo más numerosos sobre las venas, epifilo con el mismo tipo de indumento pero menos denso; venación sobresaliente en el hipofilo, 4 pares de venas laterales rectas hasta el margen. Cimas axilares abreviadas, paucifloras, ejes y pedicelo 2-3 mm long., pubescentes. Sépalos ovado-lanceolados, connados en la base, trinervados, $3 \mathrm{~mm}$ long. $x$ $1 \mathrm{~mm}$ lat., cara interna con pelos capitados diminutos dispersos, pelos estrellados en la cara externa. Pétalos membranáceos, alas de la uña recurvadas, angostas, subagudas, con pelos pequeños en la cara inferior, capucha con un pliegue profundo sobre la línea media, margen apoyado en la cara interna de los lóbulos estaminoidales, pelos dispersos en la porción distal; lámina de los pétalos filiforme, ca. $5 \mathrm{~mm}$ long., con algunos pelos cerca de la capucha, el resto glabro. Tubo estaminal glabro, membranáceo, campanulado, ca. $1 \mathrm{~mm}$ alt., anteras ditecas sobre una brevísima porción libre de filamento que es sobresaliente, estaminodios membranáceos, margen con un pequeño apículo entre dos lóbulos redondeados, cara externa de los estaminodios con tres prominen- 
cias a la altura de las anteras, las laterales algo más pequeñas que la central y ésta levemente desplazada hacia la base; cara interna de los estaminodios con una prominencia trunca. Gineceo incluido en el tubo estaminal, ovario globoso, mamilífero, estilo breve, estigma capitado, 5-lobado. Fruto leñoso, subesférico, 10-12 mm diám., disgregándose cuando maduro en 5 cocos indehiscentes, cubiertos de prominencias cónicas, apiñadas, desiguales en tamaño, finamente pubescentes.

Paratipo: BOLIVIA. Santa Cruz: prov. Nuflo de Chavez, Estancia San Miguelito, 200 km NE de la ciudad de Santa Cruz; campamento Pascana WCS Bosque ripario del río San Julián; al S del campamento, $17^{\circ} 45^{\prime} \mathrm{S} 61^{\circ} 47^{\prime} \mathrm{W}, 360 \mathrm{~m}$. Arbusto sarmentoso, 2 m; frutos secos equinados. 6-8 Jul 1995 [en fruto]. Fuentes 934 (CTES,USZ).

Especie dedicada al coleccionista, Alfredo Fuentes Claros, botánico del herbario del Museo de Historia Natural «Noel Kempff Mercado" de Santa Cruz de la Sierra, quien gentilmente me mandó el material para su estudio.

Byttneria fontis pertenece a la sección Crassipetala Cristóbal, por ser un arbusto apoyante aculeado, con frutos leñosos que se disgregan en 5 cocos indehiscentes, cubiertos de prominencias cónicas, apiñadas. Las flores tienen los estaminodios con tres prominencias en la cara externa y la capucha de la uña de los pétalos se apoya en el borde del tubo estaminal.

Dentro de la sección Crassipetala pertenece al grupo de especies constituido por $B$. microphylla Jacq., B. Aristeguieta Cristóbal, B. filipes Mart. ex K. Schum., B. Beyrichiana K. Schum. y B. Fernandesii Cristóbal, por tener pétalos y tubo estaminal membranáceos y la lámina de los pétalos muy larga y delgada que no es lo más frecuente en la sección. Sin embargo presentan otros caracteres de Crassipetala, en el caso de $B$. fontis los estaminodios tienen la cara externa con 3 prominencias y los frutos con cocos indehiscentes, caracteres que se consideran especializados dentro de la sección (Cristóbal, 1976, 63-64). B. fontis se diferencia fácilmente por una particular combinación de caracteres. A la forma exclusiva de las hojas se suma el tipo de estaminodios, pétalos, indumento y frutos.

Con $B$. fontis asciende a 26 el número de especies de la sección Crassipetala, de las cuales sólo 3 son extramericanas (Cristóbal, 1976, 1985 y 1989, Barnett \& al. 1990).

\section{Ayenia latifolia Cristóbal nov.sp.}

Fig. 2

Suffrutex rhizomatosus, ramis $10-30 \mathrm{~cm}$ altis. Folia coriacea, nitida, supra subtusque conspicue venosae, glabrae vel puberulae; laminae inferiorae et mediae ramorum ovalae vel obovalae, obtusae vel subacutae, basae cuneatae vel rotundae, inferiorae $2,6 \times 1,5 \mathrm{~cm}$, ad 3,5-1,8 $\mathrm{cm}$, mediae ca. 5,5 $\times 2,5 \mathrm{~cm}$, ad $8,2 \times 4 \mathrm{~cm}$; versus apicem oval-lanceolatae vel oblonga-lanceolatae, acutae vel subacutae, ca. $3,8 \times 1,2 \mathrm{~cm}$, ad $5 \times 1,8$. Flores diclinodioecii.

Holotipo: BRASIL. Bahia: $36 \mathrm{~km}$ SW de Correntina. En cerrado, suelo arenoso. Erecta, rizomatosa, 10-30 cm alt., hojas muy coriáceas, Iustrosas, toda la flor rojo-oscuro, brotes y aculeolos del fruto rojizos. 2.II.1963, A. Krapovickas 30173 (HUEFS). Isotipos: CTES,MBM, $\mathrm{MICH}, \mathrm{K}$.

Sufrútice erecto, $10-30 \mathrm{~cm}$ alt., rizomatoso, rizomas $2-3 \mathrm{~mm}$ diám., tallo aéreo con pelos estrellados rígidos que dejan ver la superficie de la epidermis. Estípulas lineares, rígidas, 2-5 $\mathrm{mm}$ long., vena media ancha y sobresaliente, pelos estrellados dispersos en el borde y en la cara externa, a veces en las hojas basales estípulas lanceoladas, subfalcadas, hasta de 1 $\mathrm{cm}$ long. $x$ 0,2 cm lat. Pecíolo 3-5 mm long., semiterete. Lámina coriácea, lustrosa, en las hojas proximales y mediales oval u obovada, obtusa o subaguda, con base redondeada o cuneada, en las hojas distales oval-lanceolada u oblongo-lanceolada, aguda, subaguda u obtusa, con base redondeada; las proximales promedio 2,6 x 1,5 cm (hasta 3,5 x 1,8, relación largo-ancho 1,7:1), las mediales promedio $5,5 \mathrm{x}$ $2,5 \mathrm{~cm}$ (hasta $8,2 \times 4 \mathrm{~cm}$, relación largo-ancho $2,3: 1$ ); promedio $3,8 \times 1,2 \mathrm{~cm}$ (hasta $5 \times 1,8 \mathrm{~cm}$, relación largo-ancho 3,3:1); margen aserrado, irregular, dientes progresivamente más profundos hacia el ápice, entero en el tercio o cuarto basal, venación mayor y menor sobresaliente en ambas caras, 2 pares de venas basales finas, 1 par suprabasal y 3 pares en la mitad 


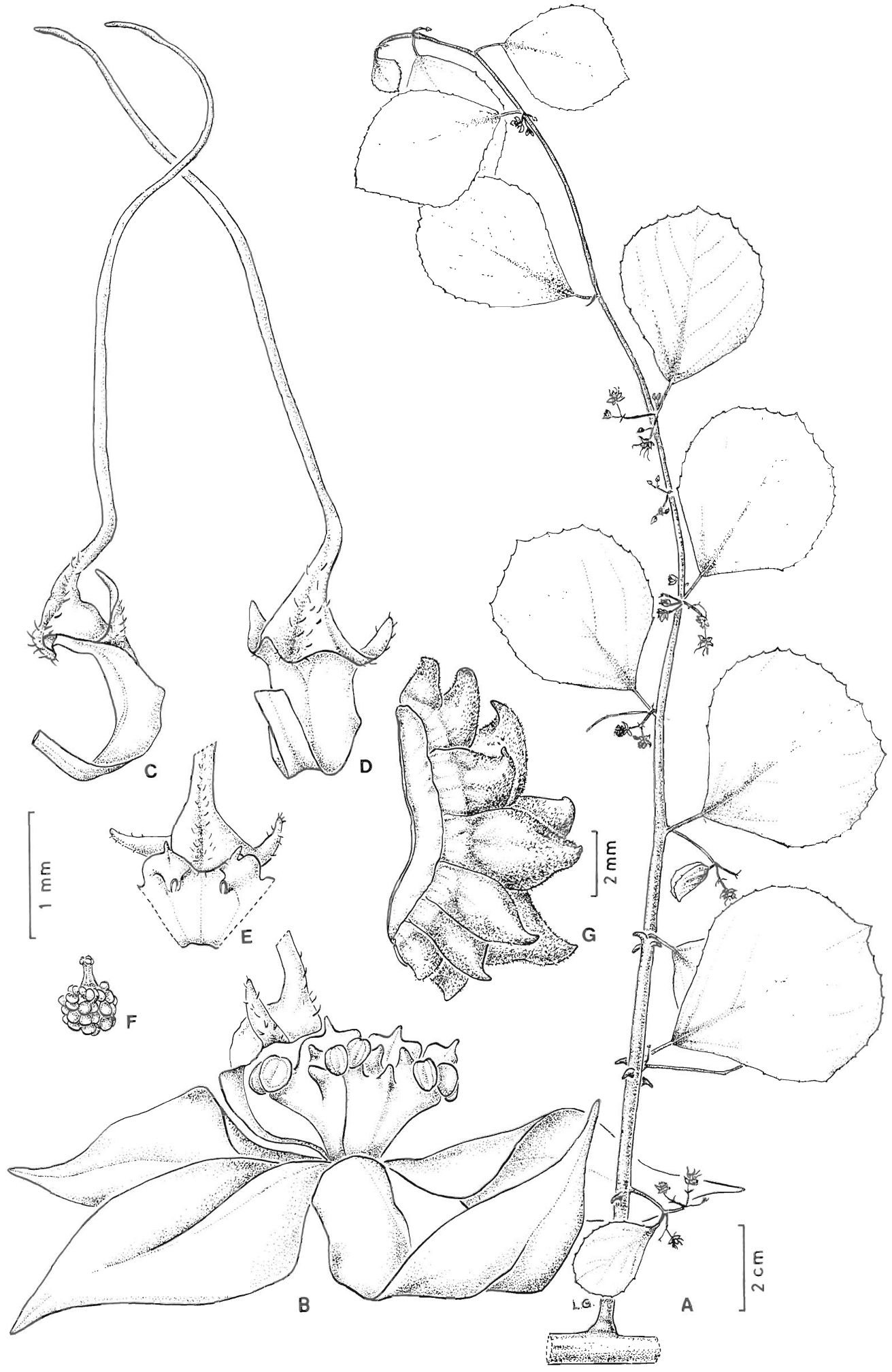

Fig. 1.Byttneria fontis: A, rama; $B$, flor en vista lateral, con la capucha de un pétalo; $C$, pétalo de perfil; $D$, pétalo, cara interna; E, cara interna de dos estaminodios y un pétalo adosado; F, gineceo; G, coco. (A-F, holotipo; G, Fuentes 934). 
distal, algo arqueadas hasta el margen; epifilo glabro o con escasos pelos estrellados rígidos en la base de la vena media o dispersos en toda la superficie, hipofilo glabro o con pelos dispersos cerca del margen o en toda la superficie. Flores totalmente rojo-oscuras, diclinas, en glomérulos axilares, densos, multifloros, a veces dispuestos desde el cuello de la planta. Brácteas escuamiformes. Pedicelo $2 \mathrm{~mm}$ long. Sépalos ovales, pelos estrellados pequeños dispersos en la cara externa, cara interna con numerosos pelos glandulares capitados. Pétalos largamente unguiculados, describiendo un semiaro, porción basal linear, porción terminal o capucha laminar, escotada en el ápice, con pelos largos en la cara externa, adosada al borde del tubo estaminal, lámina reducida a un apéndice claviforme ubicado en la cara externa de la capucha, con el extremo algo aguzado y más oscuro; flor femenina: pétalos $4 \mathrm{~mm}$ long., capucha trapezoidal, androginóforo 0,3 $\mathrm{mm}$ long., tubo estaminal urceolado, $1 \mathrm{~mm}$ long., estambres 5, con una breve porción libre de filamento, anteras tritecas, rudimentarias, estaminodios 5, recurvados, estilo exerto, estigma capitado, con 5 lóbulos conspicuos, redondeados; flor masculina: pétalos $6 \mathrm{~mm}$ long., capucha romboidal, lámina sobresaliendo del margen de la capucha, androginóforo y tubo estaminal 2,3 mm long., estaminodios cerrando la abertura del tubo estaminal, gineceo reducido, estilo breve, trunco, incluido en el tubo, lóbulos estigmáticos no desarrollados. Fruto cabizbajo, subesférico, algo complanado, 9-10 $\mathrm{mm}$ de diám., con acuieolos lineares 1-3 mm long., verdes o rojo-oscuros, pedicelo $4-5 \mathrm{~mm}$ long.; semilla negra, 4,5 mm long. x 2,5 mm lat., ovoide, rugosa, densamente tuberculada.

Paratipos: BRASIL. Bahia: Espigão Mestre, ca. $100 \mathrm{~km}$ WSW of Barreiras, elev. $760 \mathrm{~m}$; sandy campo with scattered trees and shrubs, 6.III.1972, Anderson, Stieber \& Kirkbride, Ir. 36664 (CTES, $\mathrm{MICH}, \mathrm{NY}, \mathrm{R})$. Goiás: Mun. Ponte Alta do Bom Jesus, $54 \mathrm{~km} \mathrm{~S}$ de Dianópolis y $14 \mathrm{~km}$ E del entroncamiento para Taguatinga, estrada DianópolisBarreiras, $11^{\circ} 54^{\prime} \mathrm{S} 46^{\circ} 37^{\prime} \mathrm{W}, 740 \mathrm{~m}$; cerrado muy abierto con estrato herbáceo poco denso, arena rojiza, 17.III.1982, Krapovickas, Valls \& Silva 37928 (CEN, CEPEC, CTES, NY). 20 km E de Dianópolis, Fazenda Agua Limpa, $11^{\circ} 40^{\prime} \mathrm{S} 46^{\circ} 38^{\prime} \mathrm{W}$, 17.III.1982, Krapovickas, Valls \& Silva 37867 (CEN, CTES). Rio da Prata, ca. 6 km S de Posse, elev. 800 m; subshrub ca. $25 \mathrm{~cm}$ tall, flowers purplish; sandy cerrado. 4.IV.1966, Irwin, Grear, Souza \& Reis dos Santos 14356 (NY).

Ayenia latifolia pertenece a la sección Ayenia, y dentro de ella es afín a A. angustifolia A.St.- Hil. \& Naud. Estas especies tienen en común la particularidad de poseer flores morfológicamente perfectas pero funcionalmente diclino-dioicas, carácter no conocido para otras especies del género. Se enumeran a continuación diferencias entre Ayenia iatifolia y A. angustifolia entre las cuales las del sistema subterráneo y las foliares son las de mayor peso. A. latifolia es rizomatosa, las ramas son de no más de $30 \mathrm{~cm}$ alt. y 1,5-2 $\mathrm{mm}$ diám., tiene hojas lustrosas con la venación mayor y menor muy sobresaliente en ambas caras, con una notable variación en forma y tamaño de la lámina a lo largo de las ramas, las flores son muy pequeñas, integramente rojo-oscuras y pueden estar apiñadas desde los nudos basales, las semillas son rugosas, con costillas preferentemente longitudinales. A. angustifolia posee una raíz pivotante y profunda, hasta de $1 \mathrm{~cm}$ diám., las ramas anuales son robustas hasta $4 \mathrm{~mm}$ diám. y 50-60 cm alt., llegando hasta $1 \mathrm{~m}$, y parten de un cuello leñoso. Las hojas no son lustrosas, tienen en general un indumento mucho más denso, son angustioblongas o lanceoladas y no presentan la notable variación en forma a lo largo de las ramas de $A$. latifolia. En algunas plantas de $A$. angustifolia, las hojas basales son ovales de hasta $4 \times 2 \mathrm{~cm}$, pero progresivamente se afinan hacia el ápice. Las flores son algo mayores y frecuentemente rosadas y las semillas están notablemente divididas en cuadrantes por costillas longitudinales y transversales.

En lo que se refiere a la distribución geográfica y hábitat, $A$. angustifolia vive en el centro-este de Brasil, con un área que abarca $S$ de Maranhão, Bahia, centro y norte de Minas Gerais, E de Tocantins, N de Goiás, el Distrito Federal y SE de Mato Grosso. A. latifolia posee un área incluida en la de $A$. angustifolia, reducida al E de Goiás y W de Bahia. Ambas viven en el cerrado con suelo arenoso, y A. angustifolia también en campos rupestres rocosos. En la localidad típica, A. latifolia convive con $A$. 


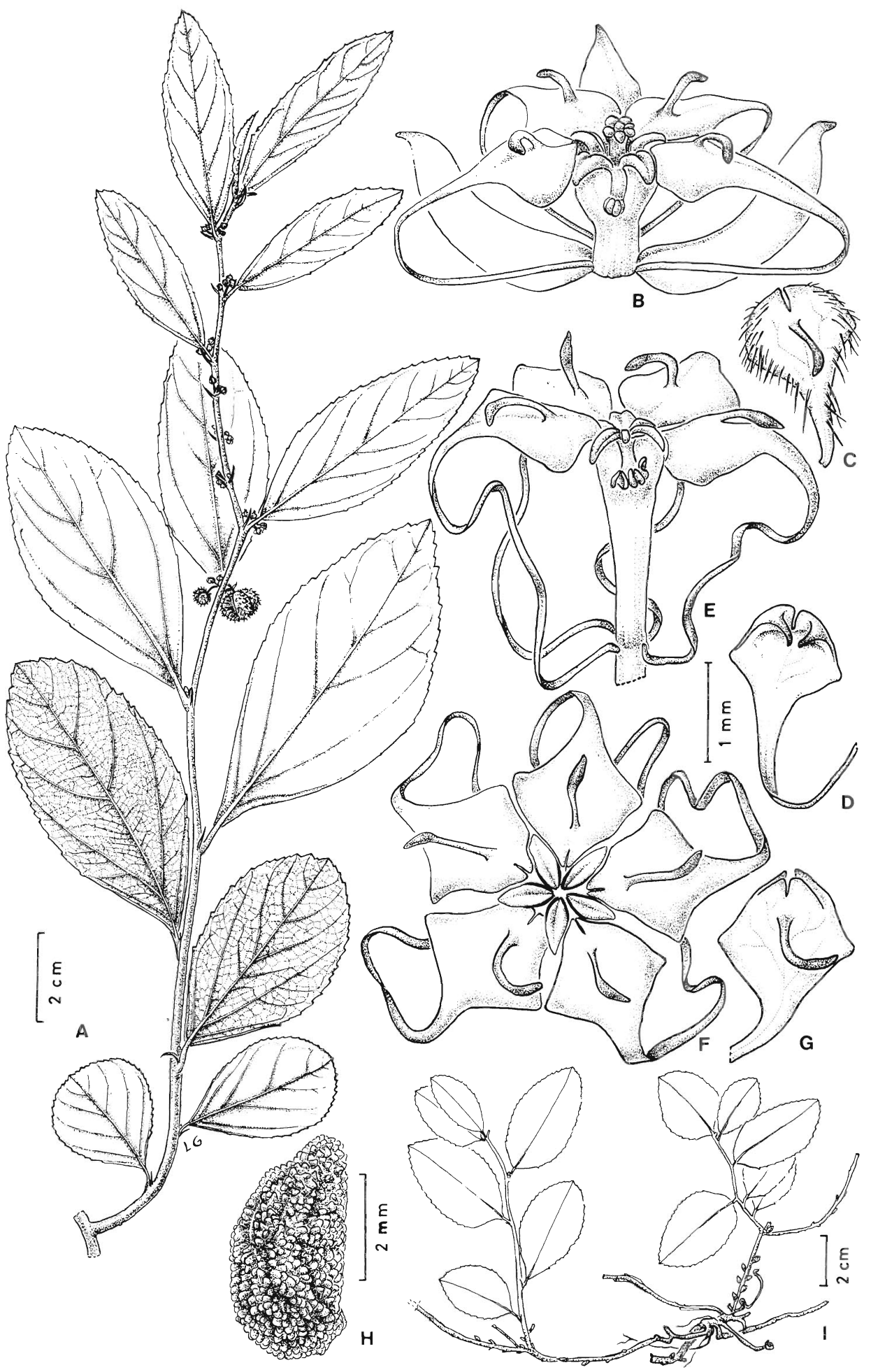

Fig. 2. Ayenia latifolia: A, rama; B, flor femenina, vista lateral (faltan un pétalo y dos sépalos); C, capucha del pétaio faltante en A, cara externa; D, capucha del mismo pétalo, cara interna; E, flor masculina (faltan un pétaì y los sépalos); F, flor masculina vista desde arriba; $G$, capucha del pétalo faltante en $E$, sin el indumento, cara externa; $H$, semilla; I, porcion de planta con el rizoma. (A-H holotipo, I, Krapovickas \& al. 37867). 
angustifolia (Krapovickas 31172) no habiéndose encontrado ejemplares intermedios. W.R. Anderson e H.S. Irwin también coleccionaron $A$. angustifolia (A. 36734 \& I. 14442 y 14492), en los mismos lugares citados para A. latifolia. El estudio de estas colecciones no dejan dudas de que se trata de entidades bien diferenciadas.

\section{Bibliografía}

BARNETT, L.C. \& L.J. DORR. 1990. A new, arborescent species of Byttneria (Sterculiaceae) from French Guiana.
Brittonia 42(4): 271-275, fig. 1.

CRISTOBAL, C.L. 1960. Revisión del género Ayenia (Sterculiaceae). Opera Lilloana 4: 1-230, 79 figs.

CRISTOBAL, C.L. 1976. Estudio taxonómico del género Byttneria Loefling (Sterculiaceae). Bonplandia 4: 1-428, 100 figs.

CRISTOBAL, C.L. 1985. Una nueva especie de Byttneria (Sterculiaceae) del Perú. Bol. Soc. Argent. Bot. 24 (1-2): 125-129, fig. 1.

CRISTOBAL, C.L. y M.M. ARBO. 1989. Byttneria caripensis (Sterculiaceae) una nueva especie de Venezuela. Bonplandia 6 (4): 125-131, figs. 1-2.

SCHUMANN, K. 1886. Sterculiaceae, en Martius, C., Fl. bras. 12 (3): 2-114, figs. 1-24. 